Article

\title{
Formulating O/W Emulsions with Plant-Based Actives: A Stability Challenge for an Effective Product
}

\author{
Alessandra Semenzato ${ }^{1, *(\mathbb{D}}$, Alessia Costantini ${ }^{2}$, Marisa Meloni ${ }^{3}$, Giada Maramaldi ${ }^{4}(\mathbb{D}$, \\ Martino Meneghin ${ }^{4}$ and Gianni Baratto ${ }^{5}$ \\ 1 Department of Pharmaceutical and Pharmacological Sciences, University of Padova, Via Francesco Marzolo \\ 5, 35131 Padova, Italy \\ 2 UniR\&D srl, Spin-Off University of Padova, Via Niccolò Tommaseo 69, 35131 Padova, Italy; \\ alessia.costantini@unired.it \\ 3 Vitroscreen srl, P.le Giulio Cesare 17, 20145 Milan, Italy; marisa.meloni@vitroscreen.com \\ 4 INDENA S.p.A., Viale Ortles 12, 20139 Milan, Italy; giada.maramaldi@indena.com (G.M.); \\ martino.meneghin@indena.com (M.M.) \\ 5 UNIFARCO S.p.A., Via Cal Longa 62, 32035 Santa Giustina (BL), Italy; gianni.baratto@unifarco.it \\ * Correspondence: alessandra.semenzato@unipd.it; Tel.: +39-049-8275356
}

Received: 6 September 2018; Accepted: 2 October 2018; Published: 9 October 2018

check for updates

\begin{abstract}
Quality, safety, and efficacy concerns added to instability, poor absorption, and the dispersion of actives are common problems while formulating plant-based cosmetics. Furthermore, a correct balance between the stability of the emulsion, the sensory profile, and the high efficacy has to be considered to formulate an effective product. In this paper, we demonstrate that rheology is a methodological tool that can be used while designing a new product. In particular, we developed an $\mathrm{O} / \mathrm{W}$ emulsion which is easy to spread on irritated skin, and that can soothe the redness and discomfort caused by the exposure to both physical and chemical irritating agents. The green active mixture consists of three natural raw materials: Bosexil ${ }^{\circledR}$, Zanthalene ${ }^{\circledR}$, and Xilogel ${ }^{\circledR}$. Each ingredient has a well-demonstrated efficacy in terms of soothing, anti-itching, and moisturizing properties respectively. Starting from the selection of a new green emulsifying system, through the analysis of the rheological properties, we obtained a stable and easy-to-apply o/w emulsion. The efficacy of the optimized product was assessed in vitro on intact and injured skin using the SkinEthic ${ }^{\mathrm{TM}}$ Reconstituted Human Epidermis (RHE) as a biological model.
\end{abstract}

Keywords: rheology; plant-based actives; emulsion design

\section{Introduction}

The development of a new successful cosmetic product must take into account, besides the technical and regulatory requirements, the marketing and consumer needs, which are continuously evolving in modern society. In fact, the R\&D departments of cosmetic companies face every day the growing demand for new product launches by the marketing divisions. Plant-based actives that have been used both in topical pharmaceutical products and in functional cosmetics for a long time for their well-documented biological effects fit very well with the current green cosmetic market trend, and can actually be considered more interesting now than in the past as cosmetic ingredients [1]. Formulating cosmetics with plant-based actives can pose technical challenges to formulators, such as the instability of the ingredient, poor absorption of actives in the outermost skin layer, dispersion problems, and quality, safety, and efficacy concerns. Most of these technical problems can be overcome using high quality standardized raw materials. However, the study 
of the interactions between the active ingredients and the vehicle components should always be included in a proper product development design, especially when high concentrations of plant-based derivatives are used to maximize the efficacy of the product. The long-term stability of a cosmetic formulation is a compulsory requirement to guarantee product safety, but the stability testing protocols are time-consuming procedures [2]; Formulators have the responsibility to prevent the failure of these testing protocols, which could even determine a delay in the product launch. As a matter of fact, the acceptance of an emulsion is strongly related to its flow properties, as well as to the physicochemical interactions that determine its inner structure. The most challenging task for cosmetic formulators is to achieve the right balance between rheological features, in terms of usability and stability of the emulsion, and the efficacy of the product in terms of the concentration of its active ingredients. In this context, rheology can play a significant role in defining and controlling the in-use performance, stability, and the usability of formulations [3]. The relevance of rheological studies as a powerful tool to objectify the skin feeling properties of cosmetics was firstly pointed out by Brummer in 1999 [4], and has been confirmed by many papers since then [5-10]. Rheology is used in more and more industries for product development and quality control. By determining the relevant rheological parameters, relationships between structure, process behavior, and final product properties can be established. The rheological curves, obtained in continuous and oscillatory conditions, provide essential information on the structure of emulsions and on their physical stability [11,12]. By considering the whole set of parameters obtained as a function of shear rate, strain, frequency, and temperature, it has been demonstrated that the risk of failure for the long-term stability procedure of cosmetic products can be preliminarily evaluated [13-15]. Starting from those concepts, we formulated a new personal care product which is easy to spread on irritated skin. In particular, it is a fluid emulsion that can soothe redness and discomfort resulting from exposure to both physical and chemical irritating agents. The green active mixture selected for the product is formed by three natural raw materials: Bosexil ${ }^{\circledR}$, Zanthalene ${ }^{\circledR}$, and Xilogel ${ }^{\circledR}$, all manufactured by INDENA ${ }^{\circledR}$. Each ingredient has well-demonstrated efficacy in terms of soothing, anti-itching, and moisturizing properties respectively. Bosexil $^{\circledR}$ is the Phytosome ${ }^{\circledR}$ form of the extract of frankincense, a perfumed resin obtained from the Indian plant Boswellia serrata, and widely used in Indian Ayurvedic medicine to counteract inflammatory conditions [16]. The extract from the resin contains different types of boswellic acids, and several studies indicate excellent efficacy by topical application [17]. The Phytosome ${ }^{\circledR}$ form improves the bioavailability of boswellic acids as an effective delivery system, and contains not less than $25 \%$ actives by HPLC, being the major component of $\beta$-boswellic acid. The Phytosome ${ }^{\circledR}$ solid dispersion contains Lecithin, a surfactant that could affect the stability of emulsions; this is an important matter to take into account during the development of a cosmetic product [18]. Zanthalene ${ }^{\circledR}$ is a $\mathrm{CO}_{2}$ extract from the fruit husks of Zanthoxylum bungeanum (commonly known as Sichuan pepper), a plant widely used in the Asian gastronomy as a spice. In vitro investigations have shown that the lipophilic extract from Z. bungeanum interacts with voltage-dependent $\mathrm{Na}^{+}$channels. Zanthalene ${ }^{\circledR}$ could, therefore, modulate thermal and tactile sensitivity, attenuating skin discomfort and itching [19]. Xilogel ${ }^{\circledR}$ is the main component obtained from the seed of the tamarind tree (Tamarindus indica). Chemically, it is a high-molecular-weight-branched polysaccharide with a cellulose-type backbone ( $\beta-(1-4)$ D-glucose) which carries xylose and galactoxylose substituents. We chose this component because of its excellent moisturizing and restructuring properties. [20] Moreover, it is a well-characterized rheological modifier, and it allows the formation of personal care products with a sensorial and moisturizing effect which is comparable to different non-green polymers like hyaluronate and xyloglucan [21]. The product development design applied in this study included rheological analyses of each cosmetic prototype, with the aim of determining the relevance of using rheology as a methodological approach to optimize cosmetic formulations, in terms of percentage of both technical and active ingredients. Starting from the selection of a new green emulsifying system, through the analysis of the rheological properties, we obtained a stable and easy-to-apply $o / w$ emulsion that may soothe discomfort and alterations of the epidermal barrier function caused by irritant agents. A finished product was industrially prepared 
based on the information obtained by this study, and the efficacy of the formulation in restoring injured tissues was assessed in vitro, using the SkinEthic ${ }^{\mathrm{TM}}$ Reconstituted Human Epidermis (RHE) as a biological model.

\section{Materials and Methods}

\subsection{Materials}

Plant-based active ingredients: Zanthalene ${ }^{\circledR}$ (Oleyl Alcohol, Zanthoxylum Bungeanum Fruit Extract); Xilogel $^{\circledR}$ (Tamarindus Indica Seed Polysaccharide) MW: 600-700 kDa; Bosexil ${ }^{\circledR}$ (Boswellia Serrata Resin Extract, Lecithin, Microcrystalline Cellulose, Silica)_Indena S.p.A. (Milan, Italy).

Vehicle ingredients: Emulium ${ }^{\circledR}$ Mellifera (Polyglyceryl-6 Distearate, Jojoba Esters, Polyglyceryl-3 Beeswax, Cetyl Alcohol)—Gattefossé; Cosmedia ${ }^{\circledR}$ SP (Sodium Polyacrylate)—BASF (Ludwigshafen, Germany).

\subsection{Emulsion Preparation (Undisclosed Formula)}

The oil phase was prepared by dispersing the emulsifier Emulium ${ }^{\circledR}$ Mellifera and the lipophile actives (Zanthalene ${ }^{\circledR}$ and Bosexil ${ }^{\circledR}$ ) in a mixture of synthetic emollients, and the phase was heated at $75{ }^{\circ} \mathrm{C}$. Xilogel ${ }^{\circledR}$ was dispersed in the water phase, which was heated at $70{ }^{\circ} \mathrm{C}$. The oil phase was added to the water phase using a Silverson L5T laboratory mixer and homogenized for 5 min at 4500 RPM.

In Table 1 , the naming of each sample with the relative concentration of Bosexil ${ }^{\circledR}$ and Cosmedia ${ }^{\circledR}$ SP is reported:

Table 1. Naming and relative concentration of Bosexil ${ }^{\circledR}$ and Cosmedia ${ }^{\circledR}$ SP of investigated samples.

\begin{tabular}{ccc}
\hline Sample & $\begin{array}{c}\text { Bosexil }^{\circledR} \\
(\% \text { w/w) }\end{array}$ & $\begin{array}{c}\text { Cosmedia }^{\circledR} \\
(\% \text { w/w) }\end{array}$ \\
\hline CX0 & 0 & 0 \\
CX0.5 & 0.5 & 0 \\
CX1 & 1 & 0 \\
CX2 & 2 & 0 \\
CX1Y0.3 & 1 & 0.3 \\
CX1Y0.5 & 1 & 0.5 \\
CX1.5Y0.3 & 1.5 & 0.3 \\
CX2Y0.3 & 2 & 0.3 \\
\hline
\end{tabular}

\subsection{Rheological Characterization}

Rheological analyses were performed in continuous and oscillatory flow conditions using a rotational rheometer, Rheoplus Anton Paar MCR 101, at fixed temperature $23 \pm 0.05{ }^{\circ} \mathrm{C}$, equipped with a cone-plate geometry CP50-1 (fixed gap 0.098 mm) (Anton Paar GmbH, A-8054 Graz, Austria).

All the measurements were taken in triplicate to ensure the reproducibility of the analyses and to validate the strength of the data. Before carrying out the rheological analysis, all samples were stored at $23{ }^{\circ} \mathrm{C}$ for at least 4 days.

The flow properties of emulsions were measured in continuous flow conditions, with a controlled shear rate test (CSR), by recording viscosity values $(\eta)$ at increasing shear rate $\left(\gamma 0.001-1000 \mathrm{~s}^{-1}\right)$.

The viscosity at rest $\left(\eta_{0}\right)$ was calculated by fitting the flow curves of the different samples, obtained in stationary conditions as a function of shear rate, with the Carreau-Yasuda model that describes the shear thinning behavior of materials [22].

The viscoelastic behavior was investigated through tests in oscillatory flow conditions (Frequency sweep). The elastic $\left(G^{\prime}\right)$ and viscous $\left(G^{\prime \prime}\right)$ moduli were measured at fixed oscillation amplitude, within the linear viscoelastic region of each material, by varying the oscillation frequency $(10-0.01 \mathrm{~Hz})$. The trends of $\mathrm{G}^{\prime}$ and $\mathrm{G}^{\prime \prime}$ as a function of frequency, measured in linear conditions, are the "mechanical spectra" of the emulsion and describe its structural properties. From this analysis, it is possible to determine the damping factor $(\tan \delta)$ that describes the ratio of $G^{\prime \prime}$ (viscous modulus) to G' (elastic 
modulus). The damping factor varies from 0 to infinity: the closer the value is to 0 , the more elastic the material, while the closer the value is to infinity, the more the material has a viscous behavior.

A temperature sweep analysis was performed using a Peltier system by varying the temperature from 23 to $45^{\circ} \mathrm{C}$ (temperature gradient of $1{ }^{\circ} \mathrm{C} / \mathrm{min} \pm 0.05^{\circ} \mathrm{C}$ ) at a fixed frequency $(1 \mathrm{~Hz}$ ) and strain $(1 \%)$ in order to investigate the dependence of the viscoelastic properties $\left(G^{\prime}, G^{\prime \prime}\right)$ of different samples on temperature.

\subsection{Optical Microscopy}

The morphology of the emulsions was evaluated using a ZEISS AXIOVERT optical microscope (Zeiss, Oberkochen, Germany) with an immersion oil objective $100 \times$.

\subsection{Stability Testing}

The stability of the products was assessed on the base of "Cosmetics Europe Guidelines on Stability Testing of Cosmetic Products 2004" [2].

The mechanical stability of the $\mathrm{O} / \mathrm{W}$ emulsions was evaluated by centrifuging $5 \mathrm{~g}$ of the sample at $4800 \mathrm{rpm}$ for $30 \mathrm{~min}$, using a Centrifuge MPW-56 (MPW MED. INSTRUMENTS, Warsaw, Poland).

The evaluation of stability, under accelerated conditions, was performed by maintaining $50 \mathrm{~g}$ of the samples at $40{ }^{\circ} \mathrm{C}$ for 3 months, using a MEMMERT UNB 300 oven (Memmert GmbH, Schwabach, Germany).

\subsection{Re-Epithelizing Efficacy on RHE with Impaired Barrier Function}

The in vitro efficacy test was performed on SkinEthic ${ }^{\mathrm{TM}}$ Reconstituted Human Epidermis (RHE) of $0.5 \mathrm{~cm}^{2}$, a fully differentiated tissue in a chemically defined medium. The model reproduces epidermal morphology, and it has been fully characterized. The RHEs were placed in an incubator at $37^{\circ} \mathrm{C}$, $5 \% \mathrm{CO}_{2}$ and saturated humidity overnight. The test started the day after the arrival; $15 \mu \mathrm{L}$ of the emulsion was directly and uniformly applied topically, on carefully dried intact RHE tissues or on RHE tissues mechanically injured by eliminating the outermost layers of stratum corneum. Tissues were kept for $3 \mathrm{~h}$ or for $6 \mathrm{~h}$ at room temperature $\left(21 \pm 2{ }^{\circ} \mathrm{C}\right)$. Saline solution was used as a reference control. After the treatment, the tissues were rinsed with saline solution and fixed in $10 \%$ formalin. Samples were included in paraffin blocks, and sections of $5 \mu \mathrm{m}$ were obtained. Slides were stained with hematoxylin and eosin. The histological samples were analyzed under light microscopy $(20 \times$ and $40 \times$ ); the overall morphology and its modification compared to the negative controls were analyzed on at least 3 sections of the same tissue.

\section{Results and Discussion}

\subsection{Rheological Measurements}

"Products that fail to deliver on expectations are doomed to failure". Nowadays there are plenty of green-active ingredients with excellent in vitro data to support claims, but often formulators are forced to adapt existing prototypes rather than employing a strategy of formulation optimization for lack of time. One of the most important challenges in formulating green cosmetics is to determine the optimal emulsion system to deliver the desired ingredient effectively to the viable epidermis via the stratum corneum. Many emulsifying agents are available on the market, and choosing the best one is a key responsibility of the formulator.

According to the increasing demand for green emulsifiers for the preparation of cosmetics, we investigated the performance of Emulium ${ }^{\circledR}$ Mellifera, an innovative sensorial emulsifier based on a patented technology that transforms and functionalizes natural waxes.

A series of emulsions, based on this emulsifier, containing the three natural ingredients were prepared, keeping constant the concentrations of Xilogel ${ }^{\circledR}$ and Zanthalene ${ }^{\circledR}$, and varying the percentage of Bosexil ${ }^{\circledR}$. In fact, the Phytosome ${ }^{\circledR}$ form, able to improve the bioavailability of boswellic acids, 
contains Lecithin, a surfactant that could affect the rheology of the emulsion and reduce its stability over time, as described in the literature. Thus, as the manufacturer's recommended range of use of $0.5-2 \%$, we compared the rheological properties of the emulsion prepared without Bosexil ${ }^{\circledR}(\mathrm{CX0})$, with the same formulations containing respectively $0.5 \%$ (CX0.5), 1\% (CX1), and 2\% (CX2) of Bosexil ${ }^{\circledR}$.

Figure 1a reports the viscosity trends in function of shear rate (Flow curves) of the four emulsions. From these graphs, a reduction of viscosity due to the addition of Bosexil ${ }^{\circledR}$ to the formulations is clearly visible. All the samples containing the active ingredient show a similar shear thinning behavior, but their flow curves are shifted one decade lower than that of the reference emulsion CX0.

By plotting the viscosity at rest $\left(\eta_{0}\right)$ values, calculated with the Carreau-Yasuda model [8], it is possible to observe the significant lowering in this parameter as a consequence of the addition of Bosexil $^{\circledR}$ (Figure 1b) to the formulation. No quantitative difference is measurable in function of the ingredient concentration, suggesting an on-off effect of Bosexil ${ }^{\circledR}$ on the viscosity of the products.

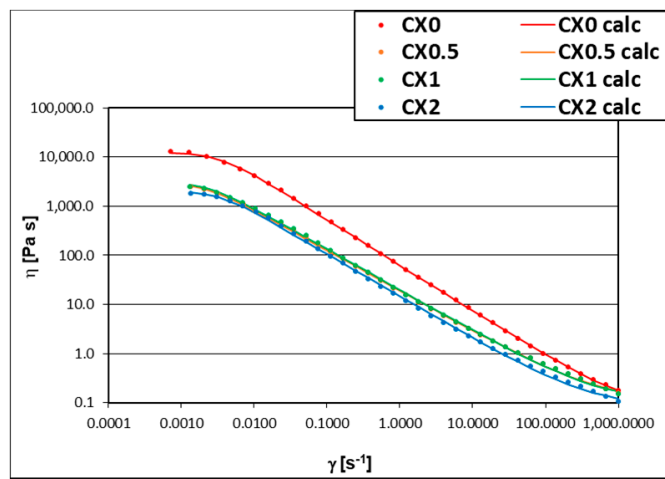

(a)

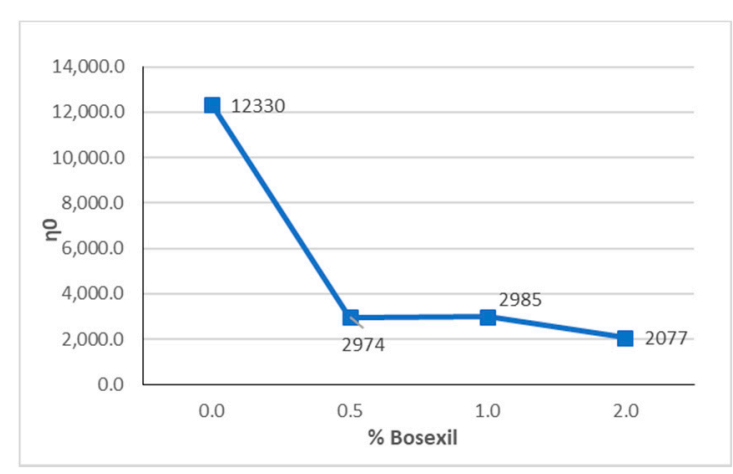

(b)

Figure 1. (a) Viscosity trends (experimental and fitted with Carreau-Yasuda model data) vs. shear rate of CX0, CX0.5, CX1, CX2 samples; (b) $\eta_{0}$ values as a function of different concentration of Bosexil ${ }^{\circledR}(0 \%$, $0.5 \%, 1 \%, 2 \%)$.

For a better understanding of the interactions between the emulsifier and the active ingredient, we also investigated the structural properties of the four emulsions in oscillatory flow conditions. When oscillatory shear measurements are performed in the linear viscoelastic regime, the elastic modulus $G^{\prime}$ (elastic response) and viscous modulus $G^{\prime \prime}$ (viscous behavior) are independent of the strain amplitude; so, by varying the oscillation frequency at fixed oscillation amplitude within the linear viscoelastic region of each material, the modulus $G^{\prime}$ (elastic modulus) and $G^{\prime \prime}$ (viscous modulus) and the viscous tangent (tan delta) can be measured. The trends of $G^{\prime}$ and $G^{\prime \prime}$ as a function of frequency, measured in linear conditions, represent the "mechanical spectra" of the emulsion and describe the structural properties of the formulation. The trends of the $G^{\prime}$ and $G^{\prime \prime}$ as a function of frequency at a fixed strain within the linear viscoelastic region (Frequency sweep) of the four emulsions are reported in Figure 2.

According to the viscosity results obtained by the flow curve measures, the addition of Bosexil ${ }^{\circledR}$ in the emulsions leads to an important reduction of the viscoelastic properties of the reference sample CX0, and a dependence on the concentration of Bosexil ${ }^{\circledR}$ can be measured.

As expected, the difference between the viscoelastic properties among the four samples are mainly quantitative: the elastic modulus $G^{\prime}$ is higher than the viscous modulus $G^{\prime \prime}$ in all the emulsions, and the moduli trends in function of frequency are quite similar, suggesting that the microstructure of the system is only weakened by the lecithin present in the natural ingredient added. 


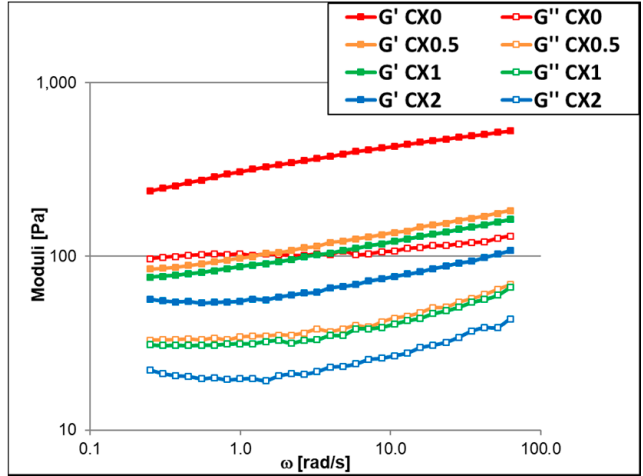

(a)

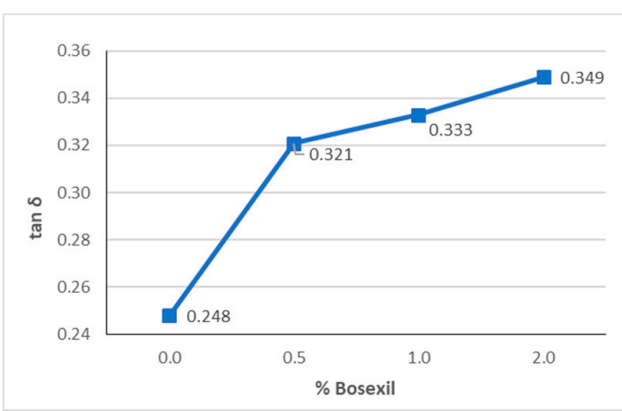

(b)

Figure 2. (a) Elastic $\left(G^{\prime}\right)$ and viscous $\left(G^{\prime \prime}\right)$ moduli in function of frequency of $C X 0, C X 0.5, C X 1, C X 2$ samples, at $1 \%$ strain; (b) tan $\delta$ values of samples (at $10 \mathrm{rad} / \mathrm{sec}$ ) prepared with different concentration of Bosexil ${ }^{\circledR}(0 \%, 0.5 \%, 1 \%, 2 \%)$.

By plotting the tan $\delta$ values of the four samples as functions of Bosexil ${ }^{\circledR}$ concentrations at $10 \mathrm{rad} / \mathrm{s}$ (Figure 2b), it is possible to understand the type and the entity of the sample microstructure variation; in fact, when $0.5 \%$ of Bosexil ${ }^{\circledR}$ is added to the formulation, a $\tan \delta$ increase of $7.3 \%$ is observed, indicating an increase of the viscous character of the formulation. Further increases of this parameter are measured as functions of the active concentration, but the extent of this effect is small, according to the hypothesis that the main agents responsible for the microstructure alteration of the emulsion could be the lecithin of the phytosome.

The rheological characterization, in continuos (flow curves) and oscillatory (frequency sweep) flow conditions, of the four emulsions was carried out at $23^{\circ} \mathrm{C}$. To further investigate the stability features of the formulations, we also performed a Temperature Sweep analysis (Figure 3). This analysis, performed in oscillatory flow conditions at fixed strain $(1 \%)$ and frequency $(1 \mathrm{~Hz})$, within the linear viscoelastic region, allowed us to investigate the dependence of the microstructure of the emulsion on temperature, and can provide useful information to evaluate how critical its stability will be over time. When the sample's structure is independent on temperature, the patterns of the elastic and viscous moduli are constant, suggesting good stability of the material over time. In personal care products, this behavior is typical of polymeric emulsifiers and of emulsion with acrylic polymers in the aqueous external phase. Green emulsions based on natural fatty acid emulsifiers and polysaccharides are usually more sensitive to temperature variations, since the melting point of fatty acids is in the range $30-50{ }^{\circ} \mathrm{C}$.

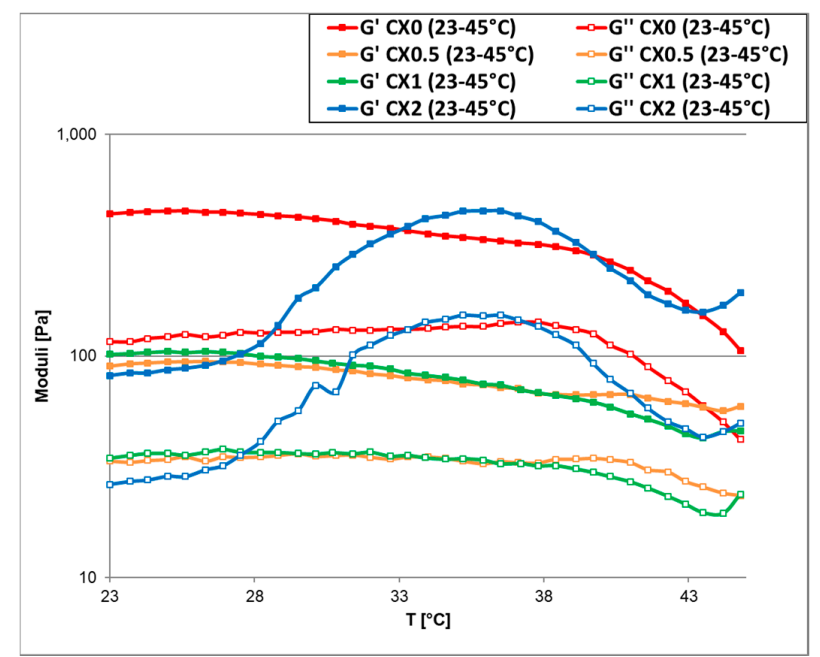

Figure 3. Trends of $G^{\prime}$ and $G^{\prime \prime}$ as a function of temperature of samples prepared with the different concentrations of Bosexil ${ }^{\circledR}(0 \%, 0.5 \%, 1 \%, 2 \%)$. 
The trends of the elastic $\left(G^{\prime}\right)$ and the viscous $\left(G^{\prime \prime}\right)$ moduli depending on the temperature in the range $23-45^{\circ} \mathrm{C}$ are shown in Figure 3. The viscoelastic properties of sample $\mathrm{CX} 2$ appear to be significantly affected by the plant-based active ingredient Bosexil ${ }^{\circledR}$ : both the viscous and the elastic moduli increase with temperature up to $36^{\circ} \mathrm{C}$ and then decrease, while in the other samples, the trends of $G^{\prime}$ and $G^{\prime \prime}$ as functions of temperature are almost linear up to $40{ }^{\circ} \mathrm{C}$, and then they slightly decrease, according to the melting point of the natural waxes of the emulsifier.

These data clearly confirm that the addition of the plant-based phytosome active Bosexil ${ }^{\circledR}$, at its maximum concentration, induces some structural changes, probably due to interference of lecithin with the emulsifier systems, as confirmed with the optical microscopy analysis by the presence of bigger droplets in the emulsion CX2 in comparison with the droplets of CX0 (Figure 4).

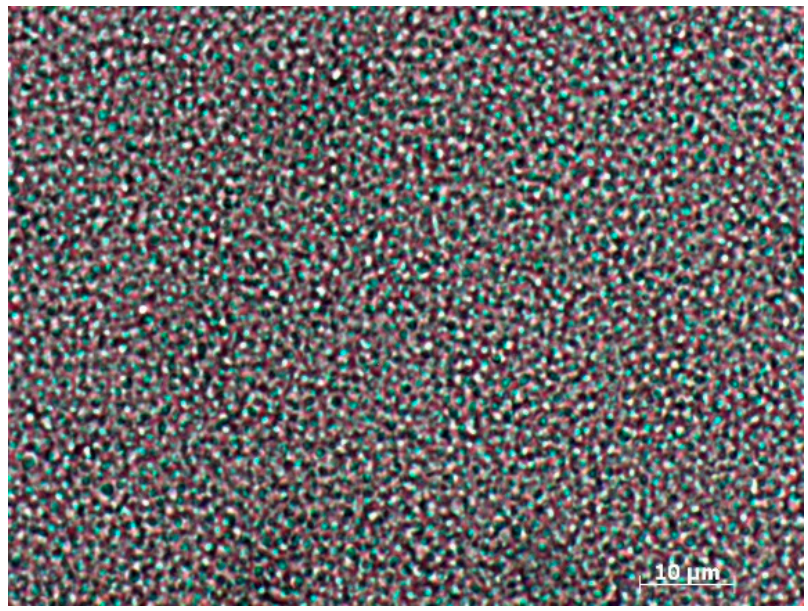

(a)

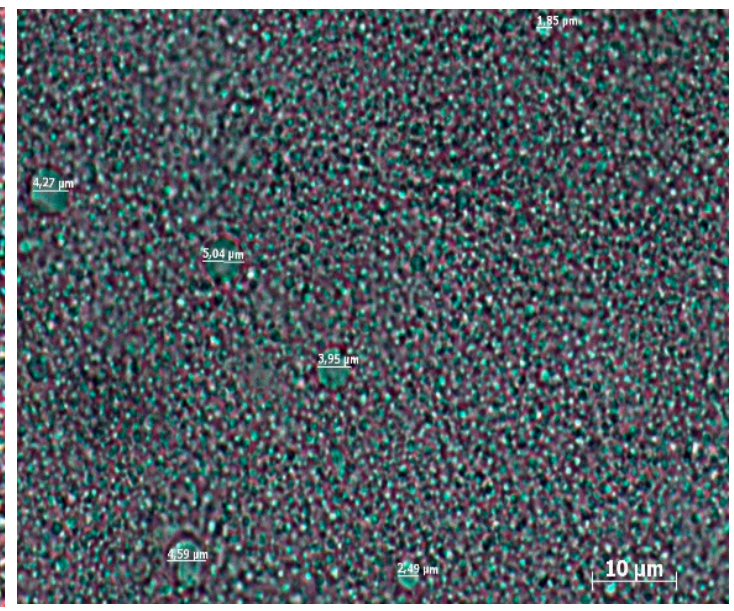

(b)

Figure 4. Optical microscopy of CX0 (a) and CX2 (b).

The stability of the four emulsions was evaluated according to the procedure reported in paragraph 2.5. All samples showed good mechanical properties and passed the centrifugation test (4800 rpm/30 min). The sample CX2 was the only one that failed the accelerated aging procedure ( 3 months storage at $40^{\circ} \mathrm{C}$ ), according to the critical issue pointed out by the temperature sweep rheological test.

It is well known that the long-term stability of emulsions can be improved by adding acrylic polymers in the external aqueous phase, with a consequent increase of the viscosity of the system at rest. With the aim of optimizing the stability of our prototypes without losing the easy-spread features of the formulation, small amounts of Cosmedia ${ }^{\circledR} \mathrm{SP}$, a thickening agent that confers also excellent sensorial aesthetics and textures to $\mathrm{O} / \mathrm{W}$ emulsions, were added to emulsion $\mathrm{CX} 1$.

In Figure 5a, the flow curves of the emulsion CX1Y0.3 and CX1Y0.5, prepared respectively with $0.3 \%$ and $0.5 \%$ of Cosmedia ${ }^{\circledR} \mathrm{SP}$, in comparison with the flow curve of emulsion $\mathrm{CX} 1$, prepared without the thickening agent, show an increase of viscosity and of shear-thinning behavior as a function of polymer's concentration, as confirmed by plotting the viscosity at rest $\left(\eta_{0}\right)$ values, calculated with the Carreau-Yasuda equation (Figure 5b).

The formula with $0.3 \%$ of the polymer was used to test the possibility of further increasing the Bosexil $^{\circledR}$ concentration in order to assess whether it was possible to obtain an even more active product.

A series of emulsions was prepared by increasing the relative amount of Bosexil ${ }^{\circledR}$ while keeping the concentration of the polymer constant at $0.3 \%$. 


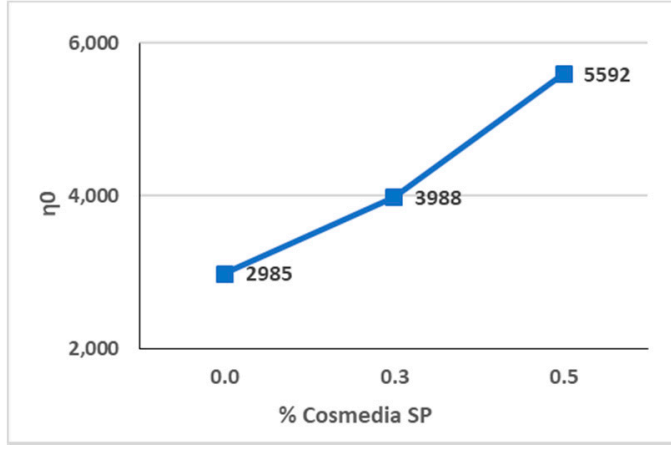

(a)

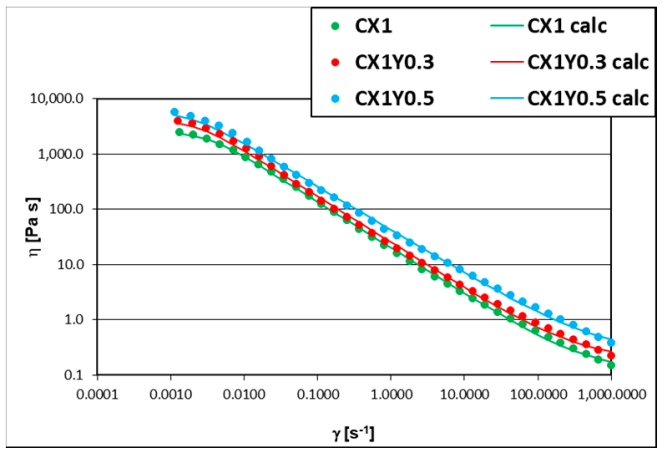

(b)

Figure 5. (a) Viscosity trends (experimental and fitted with Carreau-Yasuda model data) vs. shear rate (b) $\eta_{0}$ values of samples containing $1 \%$ of Bosexil ${ }^{\circledR}$ added with different concentrations of polymer $(0 \%, 0.3 \%, 0.5 \%)$.

The frequency and the temperature sweep analyses of the different samples are shown in Figure 6. The presence of the polymer in the external phase of the emulsion avoids the viscous-structural properties to manifest when the plant-based active ingredient is added to the formulation, as confirmed by the increase of $G^{\prime}$ and $G^{\prime \prime}$ (Figure 6a) of the emulsions prepared with 1.5\% (CX1.5Y0.3) and 2\% (CX2Y0.3) of Bosexil ${ }^{\circledR}$.

Nevertheless, the temperature sweep patterns of the samples confirm that the addition of $2 \%$ of Bosexil $^{\circledR}$ is still able to affect the emulsion structure (Figure $6 \mathrm{~b}$ ). The dependence on temperature of the elastic and viscous moduli is less evident than that of the sample CX2 (Figure 3), but an important variation of the elastic and the viscous moduli is still detectable in the range $25-35^{\circ} \mathrm{C}$.

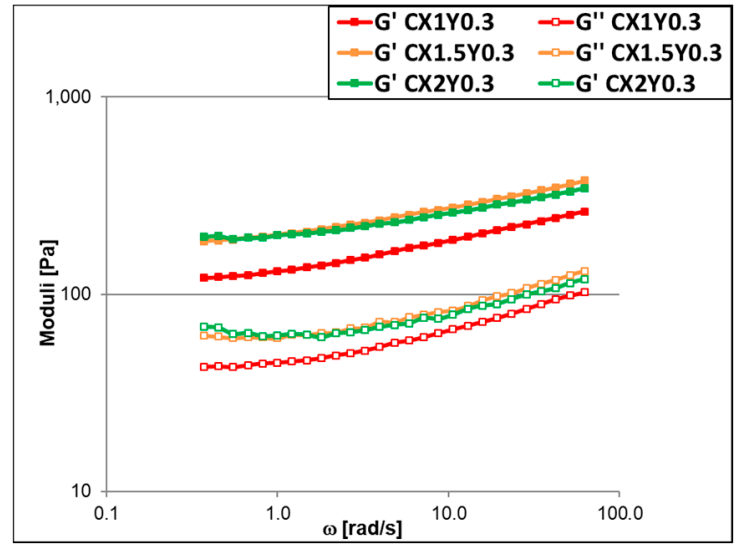

(a)

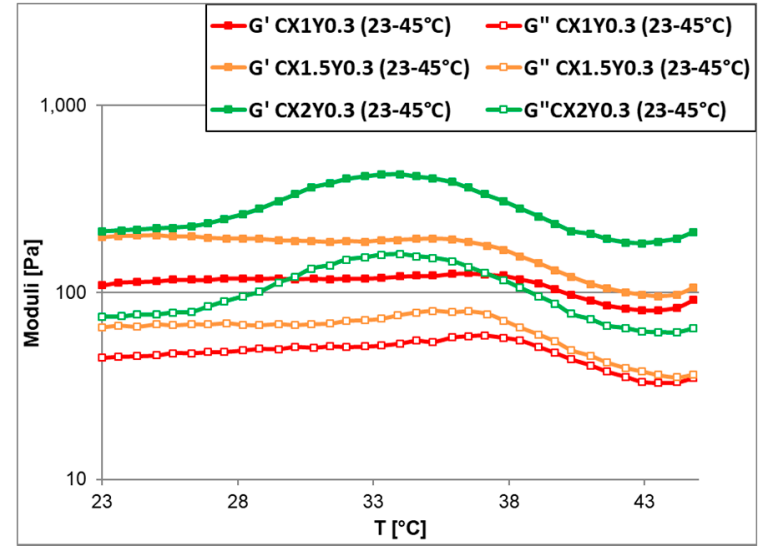

(b)

Figure 6. (a) $G^{\prime}$ and $G^{\prime \prime}$ as a function of frequency and (b) of the temperature of samples containing $0.3 \%$ of Cosmedia ${ }^{\circledR} \mathrm{SP}$ and added with different concentrations of Bosexil ${ }^{\circledR}(1 \%, 1.5 \%, 2 \%)$.

These data suggest that the two prototypes prepared with $1 \%$ and $1.5 \%$ Bosexil ${ }^{\circledR}$ and $0.3 \%$ Cosmedia ${ }^{\circledR} \mathrm{SP}$ are good candidates for becoming commercial products. As expected, these two prototypes passed the accelerated aging test while the sample containing $2 \%$ of the active ingredient showed some critical issues underlying the relevance of the rheological studies to optimize the concentration of ingredients, and to identify in advance which prototypes can be good candidates for industrial processing. 


\subsection{Re-Epithelizing Efficacy on RHE with Impaired Barrier Function}

The re-epithelizing efficacy was assessed on the intact and damaged skin, according to the procedure described in Section 2.6.

In Figure 7 the morphology of the injured reference tissues (a) is compared with the same tissue treated with the new product (b): the treatment was effective in promoting a very fast $(6 \mathrm{~h})$ recovery of the epidermal superficial architecture modified by the injury. In particular, an increased SC thickness and a more compact lipids lamellar structure is visible. These results confirm the protective and nourishing properties of the product that could be related to an active mechanism on barrier function and tight junctions structure components that could be further investigated by immunohistochemistry.

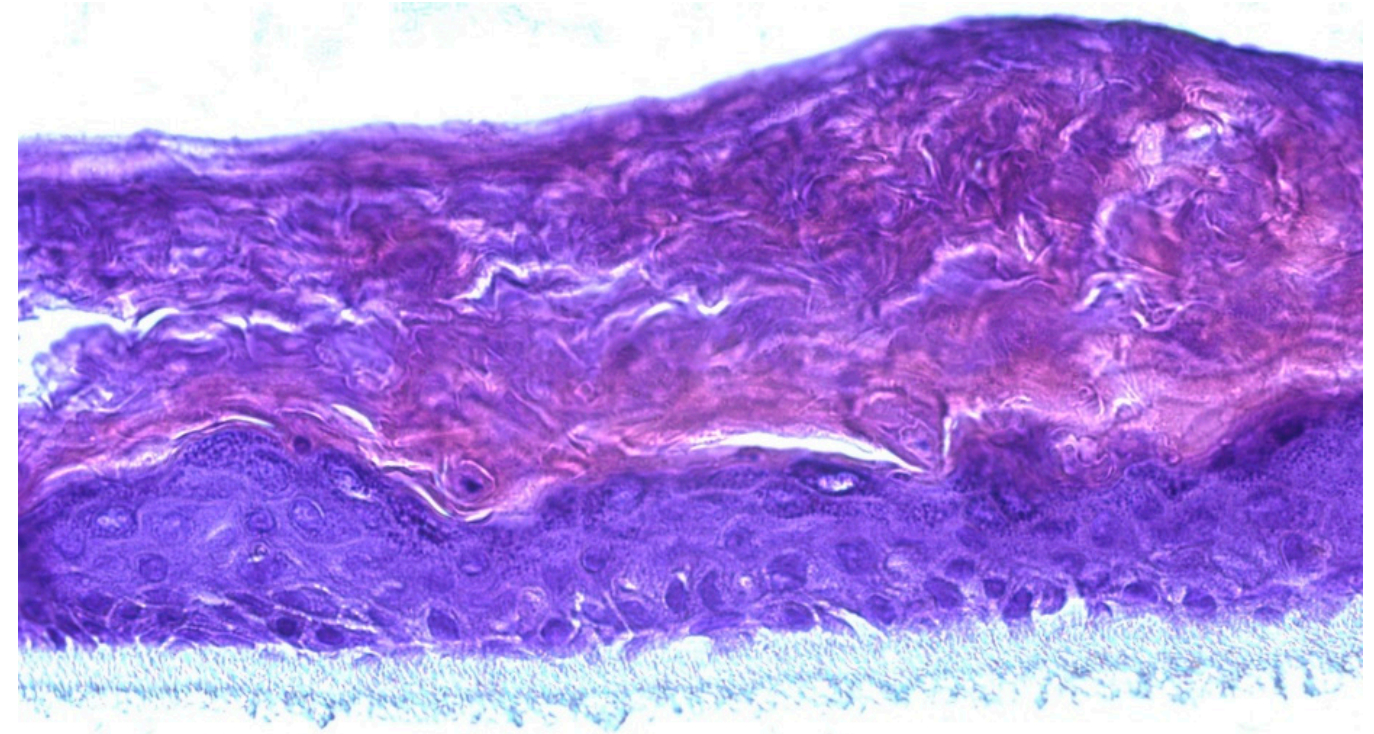

(a)

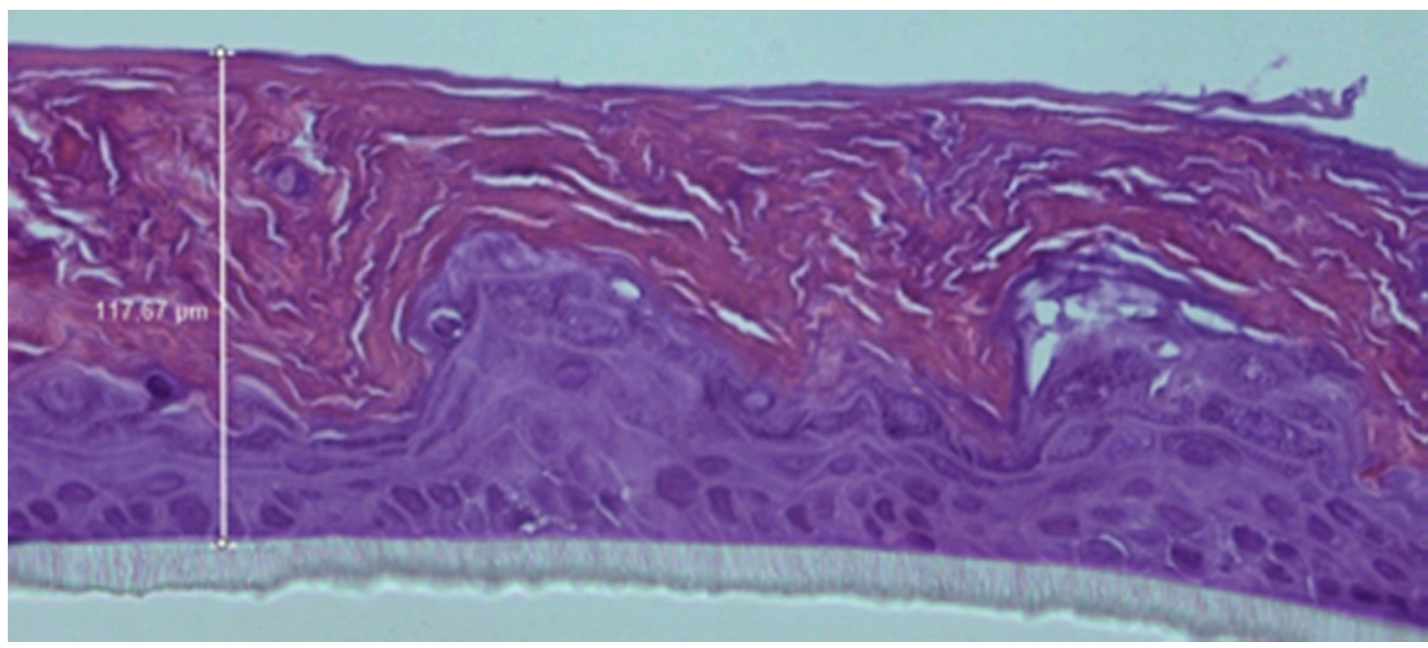

(b)

Figure 7. (a) morphology of the RHE tissue reference injured; (b) injured RHE treated with the finished product $(15 \mu \mathrm{L}, 6 \mathrm{~h})$. Enhanced SC lamellar structure and thickness are visible. 


\section{Conclusions}

This study represents a successful example of the development of a new formulation towards the fulfilment of quality requirements, in terms of good performance, stability, and retaining the acceptance criteria throughout the product's shelf-life.

The results clearly show that the rheological characterization of prototypes represents an effective tool that formulators can use in the development of a new product in order to optimize the formulation and to evaluate the product's behavior in terms of in-use performance and stability. Cosmetics are very complex multicomponent materials, and the study of their rheological behavior requires us to go beyond the measure of their viscosity in flow conditions. Each type of rheological measurement (i.e., flow curve, frequency sweep or temperature sweep etc.) can provide different information, and allows a deeper comprehension of the features of the product and of the interactions among the ingredients. This knowledge has a positive impact on all the further steps of the industrial process, from the scale up procedure to the efficacy evaluation. The in vitro recovery of the injured epidermal surface induced by the product depends on the correct combination of active and technical ingredients, which is able to enhance the SC barrier formation and to repair the injured tissue.

Author Contributions: A.S. and A.C. conceived and designed the experiments; A.C. performed the experiments and analysed the data; M.M. (Marisa Meloni) designed the in vitro analyses; G.M. and M.M. (Martino Meneghini) provided the active ingredients; G.B. provided the resources.

Funding: This research received no external funding.

Conflicts of Interest: The following study was sponsored by Unifarco S.p.A. The founding sponsors had no role in the design of the study; in the collection, analyses, or interpretation of data; in the writing of the manuscript, and in the decision to publish the results.

\section{References}

1. Amit, G.; Ashawat, M.S.; Shailendra, S.; Swarnlata, S. Phytosome: A novel approach towards functional cosmetics. J. Plant Sci. 2007, 2, 644-649.

2. Cosmetics Europe: Guidelines on Stability Testing of Cosmetic Products, 2004. Available online: https: / /www.cosmeticseurope.eu/files/5914/6407/8121/Guidelines_on_Stability_Testing_of_Cosmetics_CECTFA_-_2004.pdf (accessed on 8 October 2018).

3. Laba, D. Rheological Properties of Cosmetics and Toiletries; Marcel Dekker, Inc.: New York, NY, USA; Basel, Switzerland, 1993.

4. Brummer, R.; Godersky, S. Rheological studies to objectify sensations occurring when cosmetic emulsions are applied to the skin. Colloids Surf. A Physicochem. Eng. Asp. 1999, 152, 89-94. [CrossRef]

5. Lukic, M.; Jaksic, I.; Krstonosic, V.; Cekic, N.; Savic, S. A combined approach in characterization of an effective w/o hand cream: the influence of emollient on textural, sensorial and in vivo skin performance. Int. J. Cosmet. Sci. 2012, 2, 140-149. [CrossRef] [PubMed]

6. Lukic, M.; Jaksic, I.; Krstonosic, V.; Dokic, L.; Savic, S. Effect of Small Change in Oil Phase Composition on Rheological and Textural Properties of w/o Emulsion. J. Text. Stud. 2013, 44, 34-44. [CrossRef]

7. Gilbert, L.; Picard, C.; Savary, G.; Grisel, M. Rheological and textural characterization of cosmetic emulsions containing natural and synthetic polymers: relationships between both data. Colloids Surf. A Physiochem. Eng. Asp. 2013, 421, 150-163. [CrossRef]

8. Estanqueiro, M.; Amaral, M.H.; Sousa Lobo, J.M. Comparison between sensory and instrumental characterization of topical formulations: impact of thickening agent. Int. J. Cosmet. Sci. 2016, 38, 389-398. [CrossRef] [PubMed]

9. Calixto, M.S.; Maia Campos, P.M.B.G. Physical-Mechanical characterization of cosmetic formulations and correlation between instrumental measurements and sensorial properties. Int. J. Cosmet. Sci. 2017, 39, 527-534. [CrossRef] [PubMed]

10. Tafuro, G.; Costantini, A.; Baratto, G.; Busata, L.; Semenzato, A. Sensory texture properties of skin-care formulations: rheological and textural measurements for the characterization of finished products. In Proceedings of the 30th IFSCC Congress, Munich, Germany, 18-21 September 2018. 
11. Barnes, H.A. Rheology of emulsions-A review. Colloids Surf. A Physiochem. Eng. Asp. 1994, 91, 89-95. [CrossRef]

12. Tadros, T.F. Emulsion Formation and Stability, 1st ed.; Wiley-VCH: Weinheim, Germany, 2012.

13. Brummer, R.; Griebenow, M.; Hetzel, F.; Schlesiger, V.; Uhlmann, R. Rheological swing test to predict the temperature stability of cosmetic emulsions. In Proceedings of the 21st IFSCC Congress, Berlin, Germany, 11-14 September 2000.

14. Semenzato, A.; Zantomio, F.; Lovrecich, M.; Bettero, A.; Meloni, M. Rheology: A new approach for stability testing and formulation development. In Proceedings of the 21st IFSCC Congress, Berlin, Germany, 11-14 September 2000.

15. Semenzato, A.; Targhetta, F.; Gatti, S.; Corsi, C. Rheology as a power tool for optimizing stability of sunscreen products. In Proceedings of the XII Convegno Nazionale di Reologia, Ustica, Italy, 3-6 July 2012.

16. Togni, S.; Maramaldi, G.; Bonetta, A.; Giacomelli, L.; Di Pierro, F. Clinical evaluation of safety and efficacy of Boswellia-based cream for prevention of adjuvant radiotherapy skin damage in mammary carcinoma: A randomized placebo controlled study. Eur. Rev. Med. Pharmacol. Sci. 2015, 19, 1338-1344. [PubMed]

17. Sing, S.; Khajuria, A.; Taneja, S.C.; Johri, R.K.; Singh, J.; Qazi, G.N. Boswellic acids: A leukotriene inhibitor effective also in topical application in inflammatory disorders. Phytomedicine 2008, 15, 400-407. [CrossRef] [PubMed]

18. Traynor, M.P.; Burke, R.; Frias, J.M.; Gaston, E.; Barry-Ryan, C. Formation and stability of an oil in water emulsion containing lecithin, Xantham Gum and Sunflower Oil. Int. Food Res. J. 2013, 20, 2173-2178.

19. Artaria, C.; Maramaldi, G.; Bonfigli, A.; Rigano, L.; Appendino, G. Lifting properties of the alkamide fraction from the fruit husks of Zanthoxylum bungeanum. Int. J. Cosmet. Sci. 2011, 33, 328-333. [CrossRef] [PubMed]

20. Rao, Y.S.; Mathew, K.M. Tamarind Tamarindus indica L. research-A review. Indian J. Arecanut Spices Med. Plants 1999, 1, 127-145.

21. Semenzato, A.; Costantini, A.; Baratto, G. Green Polymers in Personal Care Products: Rheological Properties of Tamarind Seed Polysaccharide. Cosmetics 2015, 2, 1-10. [CrossRef]

22. Yasuda, K.; Armstrong, R.C.; Cohen, R.E. Shear flow properties of concentrated solutions of linear and star branched polystyrenes. Rheol. Acta 1981, 20, 163-178. [CrossRef] 